\title{
A PROPOS DE LA DISTRIBUTION DES CAS DE MALADIE ENTRE LES ASSURES ET PAR RAPPORT A LA DUREE
}

\author{
par \\ Mario Alberto Coppini \\ Rome (Italie)
}

I. Il est un fait bien connu que la technique des assurances, qu'elles soient privées ou sociales, qui versent une indemnité en cas de maladie, se base premièrement sur la connaissance des fréquences et des durées moyennes de maladie, ou bien des coefficients de morbidité. Pour résoudre des problèmes particuliers, des instruments plus raffinés sont toutefois nécessaires, savoir:

a) la distribution des assurés d'après le nombre de cas dont ils ont été frappés au cours d'une année;

b) la distribution des cas d'après la durée en jours;

c) la distribution des assurés d'après les jours de maladie dont ils ont été frappès au cours d'une annèe.

Nous avons eu la possibilité d'elaborer les fiches de 44.829 travailleurs salariés (36.134 hommes et 8.695 femmes âgés de I5 à 65 ans) résidant à Rome et assurés obligatoirement auprès de l'Institut National d'assurance-maladie (Istituto Nazionale per l'assicurazione contro le malattie - INAM), et de relever les cas de maladie qui les ont frappés au cours de l'année I960, en même temps que les durées correspondantes. ${ }^{\mathbf{1}}$ ).

Puisqu'il s'agit, comme on le verra, de données très détaillées, il nous a paru utile d'étudier les caractéristiques principales des trois distributions en question et les relations existant entre elles: l'exposé de certains résultats obtenus dans ce sens constitue l' objet de la présente note.

2. La table I recueille en premier lieu les deux distributions d'après le sexe visées à la lettre a) du paragraphe précédent, dont on obtient les indices suivants:

1) Il s'agit plus précisément de cas qui ont pris fin en I960, à l'exclusion de ceux qui n'ont pas donné lieu à annotations sur la fiche individuelle pour le motif que le pronostic formulé était inférieur à quatre jours. 
moyenne (nombre moyen de cas rapporté à tous les assurés)

Hommes Femmes

indice de répétition (nombre moyen de cas $0,502 \quad 0,534$ rapporté aux seuls assurés frappés d'un cas ou plus) variance

$\begin{array}{ll}I, 467 & I, 573 \\ 0,7129 & 0,8600\end{array}$

Nous pouvons nous demander quelle est la distribution théorique des assurés dans l'hypothèse où, une moyenne donnée ayant été fixée, l'attribution des cas de maladie aux différents individus aurait lieu au hasard: abstraction faite de la variation de la probabilité de tomber malade au cours de l'année et de la durée de la maladie, il est facile de se rendre compte que pareille distribution est celle de Poisson, puisque l'hypothèse en question correspond au schéma sur la base duquel, pour chaque assuré, grâce à une série de tirages de boules blanches et noires contenues dans une urne (les boules noires étant dans une proportion assez réduite), on attribue un cas de maladie toutes les fois que sort une boule noire.

TABLE I

Distribution des assures d'après le nombre de cas de maladie

\begin{tabular}{|c|c|c|c|c|}
\hline \multirow{3}{*}{$\begin{array}{l}\text { Nombre } \\
\text { de cas }\end{array}$} & \multicolumn{4}{|c|}{ Nombre des assurés } \\
\hline & \multicolumn{2}{|c|}{$\mathrm{H} \circ \mathrm{m} \mathrm{mes}$} & \multicolumn{2}{|c|}{$\mathrm{F}$ e $\mathrm{mm}$ es } \\
\hline & $\begin{array}{l}\text { Données } \\
\text { observées }\end{array}$ & $\begin{array}{c}\text { Données } \\
\text { ajustées (a) }\end{array}$ & $\begin{array}{l}\text { Données } \\
\text { observées }\end{array}$ & $\begin{array}{c}\text { Données } \\
\text { ajustées (a) }\end{array}$ \\
\hline o & $23.77 \mathrm{I}$ & $23 \cdot 765$ & $5.74 \mathrm{I}$ & 5.729 \\
\hline I & 8.439 & 8.398 & 1.890 & 1.902 \\
\hline 2 & 2.624 & 2.725 & 662 & 677 \\
\hline 3 & 905 & 860 & 253 & 245 \\
\hline 4 & 293 & 267 & 94 & $9 \mathrm{I}$ \\
\hline 5 & 64 & 83 & $3^{8}$ & 33 \\
\hline 6 & $3 I$ & 25 & 12 & I 2 \\
\hline 7 & 5 & 7 & 4 & 4 \\
\hline 8 & 2 & 4 & $\mathbf{I}$ & 2 \\
\hline Au total & 36.134 & 36. I 34 & 8.695 & 8.695 \\
\hline
\end{tabular}

(a) - Sur la base de la fonction (5).

Source: INAM - Travailleurs salariés de la province de Rome.

Période d'observation: Année I96o. 
On n'ignore pas que, d'après cette loi, la fréquence de ceux qui sont frappés de $r$ cas assume la forme

$$
f(r)=e^{-\lambda} \frac{\lambda^{r}}{r !} \quad\left(\sum_{0}^{\infty} f(r)=\mathrm{I}\right)
$$

En indiquant par $m$ et $s^{2}$ respectivement la moyenne et la variance des distributions examinées et par $p$ le coefficient de répétition, ${ }^{1}$ ) si les distributions susdites suivent la loi de Poisson, nous devrions avoir

$$
\begin{gathered}
\lambda=m=s^{2} \\
\rho_{p}=\frac{\lambda}{\mathrm{I}-e^{-\lambda}}=\frac{m}{\mathrm{I}-e^{-m}}
\end{gathered}
$$

$p_{p}$ étant le coefficient de répétition dans le cas de la dite loi.

On a vu que (2) n'est pas vérifié: en outre, en calculant $p_{p}$, on obtient les valeurs I,272 pour les hommes et I,29I pour les femmes, valeurs toutes deux inférieures aux valeurs observées: on peut donc conclure que la répétition plus élevée dépend du fait que celui qui est tombé malade a une probabilité plus forte d'être frappé à nouveau $^{2}$ ).

D'autre part, au cas où l'alternative opposée se vérifierait, on aurait évidemment une valeur de $p$ inférieure à celle fournie par (3) On peut donc affirmer comme indice de propension aux maladies (subséquentes) la quantité

$$
R=\frac{\rho-\rho p}{\rho p}
$$

qui assume des valeurs positives ou negatives par rapport aux deux alternatives énoncées.

1) On observera que le rapport entre la moyenne des cas et le coefficient de répétition donne la probabilité de tomber malade au moins une fois.

$\left.{ }^{2}\right)$ Il faut observer que la probabilité plus élevée de tomber malade pour une personne ayant déjà été frappée de maladie, peut dépendre du fait que la première maladie rend l'organisme plus exposé à de nouveaux événements morbides, ou bien encore de l'existence au sein du groupe observé d'une hétérogénéité a priori des individus considérés par rapport à la possibilité de tomber malade. La discrimination entre ces deux possibilités n'est pas facile à faire ni sur le plan théorique ni sur celui de la récolte des données: nous avons donc préféré nous abstenir de toute considération à cet égard. 
Pour les deux distributions examinées ici, $R$ assume la valeur de $+0,153$ pour les hommes et de $+0,218$ pour les femmes.

Pour des phénomènes analogues où l'on avait également relevé une valeur de la variance supérieure à la moyenne, GREENWOOD et YULE ${ }^{1}$ ) ont proposé, sur la base de considérations que nous ne reproduisons pas ici, la distribution de fréquences suivante

$$
\text { (5) } \left.f(r)=\left(\begin{array}{c}
p+r-\mathrm{I} \\
r
\end{array}\right)\left(\frac{a}{a+\mathrm{I}}\right) p\left(\frac{\mathrm{I}}{a+\mathrm{I}}\right)^{r}, \quad\left(\sum_{0}^{\infty}, f(r)=\mathrm{I}\right)\right)
$$

connue comme la distribution binomiale négative, pour laquelle on a

1) Cfr. M. GREENWOOD et G. U. YULE, An inquiry into the nature of frequency-distributions of multiple happenings, etc., dans "Journal of the Royal Statistical Society", I920, vol. 83 .

Il peut être utile d'observer que la distribution binomiale négative (formule (5) du texte) résulte d'un mélange de distributions de Poisson (formule (I) du texte) qu'on obtient en donnant au paramètre $\lambda(=m)$ toutes les valeurs de o à $\infty$ et en attribuant à chacune de ces distributions de Poisson un poids exprimé par la fonction

$$
g(\lambda)=\frac{a^{p}}{\Gamma(p)} e^{-a \lambda} \lambda^{p-1}
$$

c'est-â-dire qu'on obtient

$$
\int_{0}^{\infty} g(\lambda) e-\lambda \frac{\lambda r}{r !} d \lambda=\left(\begin{array}{c}
p+r-\mathrm{I} \\
r
\end{array}\right)\left(\frac{a}{a+\mathrm{I}}\right)^{p}\left(\frac{\mathrm{I}}{a+\mathrm{I}}\right)^{r} .
$$

La bonne adhérence des données observées à la fonction (5) peut donc être interprétée dans le sens que de nombreux sous-groupes coexistent au sein du groupe d'assurés observé, chacun de ces sous-groupes ayant une valeur propre de $\lambda(=m)$, c'est-à-dire son propre nombre moyen de cas de maladie auxquels on peut s'attendre pendant la période annuelle prise en

\begin{tabular}{|c|c|c|c|c|c|}
\hline$\lambda(=m)$ & Hommes & Femmes & $\lambda(=m)$ & Hommes & Femmes \\
\hline $\mathrm{o}-\mathrm{O}, \mathrm{I}$ & I.597 & I.5I 3 & $I, 4-I, 6$ & I95 & $28 I$ \\
\hline $0, I-0,2$ & I. 445 & I. 285 & $\mathrm{I}, 6-\mathrm{I}, 8$ & 126 & 203 \\
\hline $0,2-0,3$ & I. 248 & 1.090 & $1,8-2,0$ & 80 & I 45 \\
\hline $0,3-0,4$ & 1.048 & 925 & $2,0-2,5$ & 97 & 211 \\
\hline $0,4-0,5$ & 872 & 785 & $2,5-3,0$ & $3 I$ & 92 \\
\hline $0,5-0,6$ & 710 & 666 & $3,0-3,5$ & I I & 4I \\
\hline $0,6 \longrightarrow 0,7$ & $5^{89}$ & $5^{66}$ & $3,5-4,0$ & 3 & I8 \\
\hline $0,7-0,8$ & 479 & 479 & $4,0-4,5$ & $\mathbf{I}$ & 8 \\
\hline $0,8-0,9$ & $3^{88}$ & 408 & $4,5-5,0$ & I & 3 \\
\hline $0,9-\mathbf{1}, 0$ & 317 & 345 & $5,0-5,5$ & & 2 \\
\hline $\mathrm{I}, \mathrm{O}-\mathrm{I}, 2$ & 460 & 542 & $5,5-6,0$ & & $\mathbf{I}$ \\
\hline $\mathbf{I}, 2-\mathbf{I}, 4$ & 302 & $39 I$ & & 10.000 & 10.000 \\
\hline
\end{tabular}
considération.

Les valeurs de la fonction $g(\lambda)$ ont résulté être les suivants 
(6)

$$
\begin{gathered}
m=\frac{p}{a} \\
s^{2}=\frac{p}{a}+\frac{p}{a^{2}}
\end{gathered}
$$

En déterminant $a$ et $p$ sur la base des (6) et (7) et des valeurs observées de $m$ et de $s^{2}$, on a obtenu

$\begin{array}{ccc} & \text { Hommes } & \text { Femmes } \\ a & 2,377 & \text { I,64I } \\ p & \mathrm{I}, \mathrm{I} 93 & 0,877\end{array}$

Les distributions théoriques ainsi déterminées, qui sont également exposées dans la Table $\mathrm{I}$, constituent un excellent ajustement de celles déduites de l'expérience.

On peut observer enfin que pour la distribution (5) on a, en rappelant (6) et $(7)$,

$$
\rho=\frac{m}{I-\left(\frac{s^{2}}{m}\right)^{-p}}
$$

3. Les distributions des cas observés par rapport à la durée sont reproduites en synthèse dans la Table 2 et en détail dans les tableaux I et II annexés à la présente note: dans ces derniers documents, les cas sont distingués d'après les deux critères suivants:

a) l'ordre dans lequel ils ont eu lieu vis-à-vis de l'assuré;

b) leur multiplicité toujours vis-à-vis de l'assuré (c'est-à-dire le fait de se référer à des assurés frappés d'un seul cas, de deux seuls cas, etc.).

Nous nous sommes demandés si ces classifications excercent ou non une influence sur la distribution des cas par durée. Après avoir effectué l'analyse de la variance, les résultats suivants ont été obtenus.

\section{DISTRIBUTIONS D'APRES L'ORDRE}

\begin{tabular}{lcc} 
& \multicolumn{2}{c}{ Hommes } \\
Variance entre les moyennes & 608,62 & $\begin{array}{c}\text { Degrés de } \\
\text { liberté }\end{array}$ \\
Variance résiduelle & 648,05 & 4 \\
Variance globale & $648, \mathrm{I} 26$ \\
rapport entre les variances $=\mathrm{I}, 06$ & $\mathrm{~F} 5 \%=5,63$ & I8.I30
\end{tabular}




\begin{tabular}{lrr} 
& \multicolumn{2}{c}{ Femmes } \\
& Variances & $\begin{array}{c}\text { Degrés de } \\
\text { liberté }\end{array}$ \\
Variance entre les moyennes & 386,66 & 4 \\
Variance résiduelle & 468,53 & 4.642 \\
Variance globale & 468,46 & 4.646 \\
rapport entre les variances $=\mathrm{I}, 2 \mathrm{I}$ & $\mathrm{F} 5 \%=5,63$.
\end{tabular}

\section{Distributions D'APRES LA MULTIPLICITE}

\begin{tabular}{lrr} 
& \multicolumn{2}{c}{ Hommes } \\
& Variances & $\begin{array}{c}\text { Degrés de } \\
\text { liberté }\end{array}$ \\
Variance entre les moyennes & $3.110,34$ & 4 \\
Variance résiduelle & 647,50 & I8. 226 \\
Variance globale & 648,04 & I8.130
\end{tabular}

rapport entre les variances $=4,80 \quad$ F $5 \%=2,37$

$$
\text { Femmes }
$$

Variances
libertés de

Variance entre les moyennes

Variance résiduelle

372,94

$468,54 \quad 4.642$

Variance globale

$468,46 \quad 4.646$

rapport entre les variances $=1,26 \quad$ F $5 \%=5,63$

Comme on peut le constater, une seule classification résulte être significativè, et précisément celle d'après la multiplicité pour le sexe masculin.

Les deux distributions globales ont les moyennes et variances suivantes:

durée moyenne (en jours)

$\begin{array}{cc}\text { Hommes } & \text { Femmes } \\ \text { I7,45 } & \text { I } 4,6 \text { I } \\ 648, \text { II } & 468,29\end{array}$

Au moyen de ces données, on peut se proposer de vérifier quelles sont les moyennes de chaque distribution des tableaux I et II qui diffèrent d'une manière significative des durées moyennes globales, afin d'approfondir les résultats obtenus par l'analyse des variances. Dans ce but, ayant indiqué par $A$ et $\sigma^{2}$ respectivement la moyenne 
et la variance des distributions globales, par $N$ le nombre global des observations et par $n$ le nombre des observations contenues dans une distribution générique partielle, nous avons calculé les quantités $2 \sigma \sqrt{\frac{\overline{N-n}}{(N-\mathrm{I}) n}}$ et nous avons vérifiési les différences entre la moyenne globale et les moyennes de chaque distribution sont supérieures en valeur absolue aux intervalles de confiance susdits. Les résultats sont les suivants:

Distributions d'après l'ordre

\begin{tabular}{|c|c|c|c|c|c|c|}
\hline & \multicolumn{3}{|c|}{ Hom mes } & \multicolumn{3}{|c|}{ Fem mes } \\
\hline & Moyennes & $\begin{array}{c}\text { Différence } \\
\text { avec la } \\
\text { moyenne } \\
\text { générale }\end{array}$ & $\begin{array}{c}\text { Intervalle } \\
\text { de } \\
\text { confiance }\end{array}$ & Moyennes & $\begin{array}{l}\text { Différence } \\
\text { avec la } \\
\text { moyenne } \\
\text { générale }\end{array}$ & $\begin{array}{c}\text { Intervalle } \\
\text { de } \\
\text { confiance }\end{array}$ \\
\hline premiers cas & $\mathrm{I} 7,4^{\circ}$ & $-0,05$ & 0,26 & 14,66 & $+0,05$ & 0,48 \\
\hline deuxièmes cas & 17,72 & $+0,27$ & 0,72 & 14,88 & $+0,27$ & $\mathrm{I}, \mathrm{I} 7$ \\
\hline troisièmes cas & 17,27 & $-0,18$ & $I, 36$ & 14,66 & $+0,05$ & 2,06 \\
\hline quatrièmes cas & I 8,27 & $+0,82$ & 2,53 & I I, $4^{8}$ & $-3,13$ & 3,49 \\
\hline dres plus élevés & 13,95 & $-3,5^{\circ}$ & 4,15 & I 4,57 & $-0,04$ & 4,86 \\
\hline
\end{tabular}

Distributions d'après la multiplicité

\begin{tabular}{l|c|c|c|c|c|c}
\hline & \multicolumn{3}{|c|}{ Ho m m es } & \multicolumn{3}{c}{ F e m m e s } \\
\hline & Moyennes & $\begin{array}{c}\text { Différence } \\
\text { avec la } \\
\text { moyenne } \\
\text { générale }\end{array}$ & $\begin{array}{c}\text { Intervalle } \\
\text { de } \\
\text { confiance }\end{array}$ & Moyennes & $\begin{array}{c}\text { Différence } \\
\text { avec la } \\
\text { moyenne } \\
\text { générale }\end{array}$ & $\begin{array}{c}\text { Intervalle } \\
\text { de } \\
\text { confiance }\end{array}$ \\
\hline cas uniques & $\mathbf{1 7 , 5 2}$ & $+0,07$ & 0,41 & 14,80 & $+0,19$ & 0,77 \\
cas doubles & 17,70 & $+0,25$ & 0,59 & 15,12 & $+0,51$ & 1,01 \\
cas triples \\
cas quadruples \\
$\begin{array}{l}\text { cas quintuples } \\
\text { et cas d'une } \\
\text { multiplicité } \\
\text { plus élevée }\end{array}$ & 17,84 & $+0,39$ & 0,90 & 14,29 & $-0,32$ & 1,44 \\
\hline
\end{tabular}


Comme on peut le constater, seule la moyenne de la distribution relative au sexe masculin visant les cas quintuples et d'une multiplicité plus élevée résulte être significative.

On peut ainsi conclure que, sauf cas exceptionnels, ni l'ordre ni la multiplicité influent sur la distribution par durées.

Pour les cas doubles on a en outre élaboré au moyen du matériel original les tables à double entrée des assurés classifiés par rapport aux durées des deux cas, et on a obtenu les coefficients de corrélation suivants:

$$
\begin{array}{ll}
\text { pour les hommes } & +0, \mathrm{I} 39 \\
\text { pour les femmes } & +0, \mathrm{I} 2 \mathrm{I}
\end{array}
$$

Les valeurs assez modestes de ces coefficients permettent d'affirmer que, tout au moins pour les cas doubles, la durée du second cas résulte être presque indépendante de celle du premier cas.

TABLE 2

\begin{tabular}{|c|c|c|c|c|c|c|c|c|}
\hline \multirow{3}{*}{$\begin{array}{l}\text { Durée } \\
\text { (en } \\
\text { jours) }\end{array}$} & \multicolumn{4}{|c|}{ Hom mes } & \multicolumn{4}{|c|}{$\mathrm{Fem} \mathrm{mes}$} \\
\hline & \multirow[b]{2}{*}{$\begin{array}{c}\text { Données } \\
\text { obser- } \\
\text { vées }\end{array}$} & \multicolumn{3}{|c|}{ Données ajustées (a) } & \multirow{2}{*}{$\begin{array}{c}\text { Données } \\
\text { obser- } \\
\text { vées }\end{array}$} & \multicolumn{3}{|c|}{ Données ajustées (a) } \\
\hline & & $\begin{array}{l}\text { distri- } \\
\text { bution } \\
\text { globale }\end{array}$ & \multicolumn{2}{|c|}{$\begin{array}{c}\text { distributions } \\
\text { partielles }\end{array}$} & & $\begin{array}{l}\text { distri- } \\
\text { bution } \\
\text { globale }\end{array}$ & \multicolumn{2}{|c|}{$\begin{array}{c}\text { distributions } \\
\text { partielles }\end{array}$} \\
\hline$I-5$ & 3.237 & $3 \cdot 37^{2}$ & $3 \cdot 372$ & & 3.889 & 3.822 & 3.822 & \\
\hline 6 - I0 & 2.484 & 2.349 & 2.349 & & $2.31 \mathrm{I}$ & 2.380 & $2.37^{8}$ & 2 \\
\hline II - I 5 & I. $25^{\circ}$ & I.226 & I.225 & I & I. 192 & I. 167 & I. 130 & 37 \\
\hline $16-20$ & $7 \times 2$ & 608 & 595 & I 3 & 657 & 623 & 492 & $13 \mathrm{I}$ \\
\hline $2 \mathrm{I}-3^{\circ}$ & $86 I$ & $5^{67}$ & 402 & 165 & 824 & 722 & 288 & 434 \\
\hline $3 I-40$ & $45 \mathrm{I}$ & 423 & $8 I$ & 342 & 383 & $44^{8}$ & 47 & 401 \\
\hline $4 I-60$ & 432 & 686 & I 8 & 668 & 364 & 466 & 8 & $45^{8}$ \\
\hline $6 r-90$ & 298 & 486 & $\mathbf{I}$ & 485 & 187 & 242 & & 242 \\
\hline $9 I-120$ & 127 & I 67 & & I 67 & I03 & 74 & & 74 \\
\hline $121-15^{\circ}$ & 62 & 64 & & 64 & 45 & 28 & & 28 \\
\hline $\begin{array}{l}\text { Au délà de } \\
\text { I } 50 \text { jours }\end{array}$ & 86 & 52 & & 52 & 45 & 28 & & 28 \\
\hline & 10.000 & 10.000 & 8.043 & 1.957 & 10.000 & I0.000 & 8.165 & I. 835 \\
\hline
\end{tabular}

Distribution des cas de maladie d'après la durée

(a) Sur la base de la fonction (9) et de ses composantes. Source: INAM Travailleurs salariés de la province de Rome. Période d'observation: Année 1960. 
Il a été procédé enfin à l'ajustement de la distribution de la Table 2 avec la fonction suivante, proposée dans un de mes travaux antérieurs ${ }^{1}$ )

(9) $\varphi(u)=k_{1} \varphi_{1}(u)+k_{2} \varphi_{2}(u)=k_{1} \frac{\mathrm{I}}{a_{1}^{p_{1}+1} \Gamma\left(p_{1}+\mathrm{I}\right)} u^{p_{1}} e^{-\frac{u}{a_{1}}}+$

$$
+k_{2} \frac{a_{2}^{p_{2}-1}}{\Gamma\left(p_{2}-\mathrm{I}\right)} u^{-p_{2}} e^{-\frac{a_{2}}{u}} ;\left(\int_{0}^{\infty} \varphi(u) d u=\mathrm{I} ; k_{1}+k_{2}=\mathrm{I}\right)
$$

qui décompose la distribution globale en deux distributions partielles, fournies par le premier et le deuxième terme de la fonction (9), respectivement pour les maladies à élimination (de l'état morbide) rapide et pour les maladies à élimination lente.

Les valeurs des paramètres obtenus sont les suivantes:

$\begin{array}{ccc} & \text { Hommes } & \text { Femmes } \\ k_{1} & 0,804 & 0,8 \mathrm{I} 6 \\ a_{1} & 5,60 & 5,00 \\ p_{1} & 0,50 & 0,50 \\ k_{2} & 0, \mathrm{I} 96 & 0,184 \\ a_{2} & 248,9 \mathrm{I} & \mathrm{I} 39,68 \\ p_{2} & 6,00 & 5,00\end{array}$

En indiquant par $A_{1}, A_{2}, \sigma_{1}^{2}, \sigma_{2}^{2}$, respectivement les moyennes et les variances de la fonction $\varphi_{1}(u)$ et de la fonction $\varphi_{2}(u)$ on obtient en outre:

$\begin{array}{lrr} & \text { Hommes } & \text { Femmes } \\ A_{1} & 8,40 & 7,50 \\ A_{2} & 62,23 & 46,56 \\ \sigma_{1}^{2} & 47,04 & 37,50 \\ \sigma_{2}^{2} & 1.290,86 & 1.083,92\end{array}$

4. Etablissons maintenant la relation intervenant entre la distribution des assurés d'après le nombre de jours de maladie que nous indiquerons ci-après par $\psi(u)$ et celles des assurés d'après le nombre des cas et des cas d'après la durée.

1) Cfr. M. A. Coppini, Reduction factors and sickness distribution by duration, dans "Transactions of the First International Conference of Social Security Actuaries and Statisticians", Bruxelles, novembre 1956 - ISSA. Nous renvoyons le lecteur à ce travail pour la méthode utilisée dans la détermination des paramètres. 
En attendant, il est évident qu'en indiquant par $\psi_{1}(u), \psi_{2}(u)$, $\ldots, \psi_{r}(u) \ldots$ les distributions analogues des assurés qui ont été frappés respectivement de $I, 2, \ldots r \ldots$ cas, on a

$$
\begin{array}{r}
\psi(u)=\bar{f}(\mathrm{I}) \psi_{1}(u)+\bar{f}(2) \psi_{2}(u)+\ldots+\bar{f}(r) \psi_{r}(u)+\ldots \\
\left(\int_{0}^{\infty} \psi(u) d u=\mathrm{I} ; \int_{0}^{\infty} \psi_{r}(u) d u=\mathrm{I}\right)
\end{array}
$$

où on a posé

$$
\varphi_{21}(u) * \varphi_{22}(u)=\bar{f}(r)=\frac{f(r)}{\mathrm{I}-\bar{f}(0)}
$$

Indiquons par $\varphi_{11}(u)$ la distribution des cas de maladie uniques, par $\varphi_{21}(u)$ et $\varphi_{22}(u)$ respectivement la distribution du premier et du second des cas doubles, par $\varphi_{r_{1}}(u) \varphi_{r_{2}}(u) \ldots \varphi_{r r}(u)$ respectivement la distribution du premier, du deuxième, ... du rème cas des cas $r$ uples, etc. ${ }^{\mathbf{1}}$ )

\section{C'est évidemment}

$$
\psi_{1}(u)=\varphi_{11}(u) .
$$

D'autre part, supposé que le nombre de jours de maladie qui frappe un assuré lequel a subi deux cas de maladie puisse être considéré comme la somme de deux variables casuels indépendants (qu'on se rappelle la légère corrélation verifiée au paragraphe 3), on obtient comme on le sait

$$
\psi_{2}(u)=\varphi_{21}(u) * \varphi_{22}(u)
$$

en ayant posé

$$
\text { (I4) } \varphi_{21}(u) * \varphi_{22}\left(u \left(=\int_{0}^{\infty} \varphi_{21}(u-t) \varphi_{22}(t) d t\right.\right.
$$

En raisonnant d'une manière analogue pour les cas triples, $\ldots r$ uples etc., on obtient en définitive

$$
\begin{array}{r}
\left(\text { I5) } \psi(u)=\bar{f}(\mathrm{I}) \varphi_{11}(u)+\bar{f}(2)\left[\varphi_{21}(u) * \varphi_{22}(u)\right]+\ldots\right. \\
\ldots+f(r)\left[\varphi_{r_{1}}(u) * \varphi_{r_{2}}(u) * \ldots * \varphi_{r r}(u)\right]+\ldots
\end{array}
$$

1) On se rappellera le sens de cas uniques, doubles, triples, ... défini au paragraphe précédent. 
$\mathrm{Si}$ on introduit encore l'hypothèse d'après laquelle toutes les fonctions $\varphi_{\text {vs }}(u)$ peuvent être remplacées, à des fins pratiques, par la fonction $\varphi(u)$, de même qu'il a été aussi vérifié au paragraphe 3 , nous avons enfin

$$
\begin{aligned}
\psi(u) & =\bar{f}(x) \varphi(u)+\bar{f}(2)[\varphi(u) * \varphi(u)]+\ldots+ \\
& +\bar{f}(r)[\varphi(u) * \varphi(u) * \ldots * \varphi(u)]+\ldots \quad r \text { facteurs }
\end{aligned}
$$

La moyenne $\bar{A}$ et la variance $\bar{\sigma}^{2}$ de la distribution composée $\psi(u)$ s'obtiennent très facilement: en supposant connues les moyennes $\bar{A}_{1}, \bar{A}_{2}, \ldots, \bar{A}_{r}, \ldots$ et les variances $\tilde{\sigma}_{1}^{2}, \bar{\sigma}_{2}^{2}, \ldots, \bar{\sigma}_{r}^{2} \ldots$ des distributions composantes $\psi_{1}(u), \psi_{2}(u), \ldots, \psi_{r}(u) \ldots$, on obtient en effet

$$
\begin{gathered}
\bar{A}=\Sigma_{r} f(r) \bar{A}_{r} \\
\bar{\sigma}^{2}=\Sigma \bar{f}(r) \bar{\sigma}_{r}^{2}+\Sigma_{r s} \bar{f}(r) \bar{f}(s)\left(\bar{A}_{r}-\bar{A}_{s}\right)^{2},
\end{gathered}
$$

où le second membre de la somme s'entend étendu à toutes les combinaisons possibles des indices $r$ et $\left.s .{ }^{1}\right)$ En admettant l'hypothèse d'indépendance sur laquelle (I3) est basé, ainsi que l'hypothèse d'égalité que nous a mené à (I6), nous obtenons en particulier

$$
\begin{aligned}
& \bar{A}_{r}=r \bar{A} \\
& \bar{\sigma}_{r}^{2}=r \sigma^{2}
\end{aligned}
$$

Si on se souvient que

$$
\rho=\Sigma_{\gamma} f(r) r
$$

et on pose

$$
\bar{s}^{2}=\Sigma_{r s} f(r) \bar{f}(s)(r-s)^{2},
$$

en observant que la quantité correspondant au second membre de l'égalité est la moitié du carré de la différence moyenne quadratique avec répétition relative à la distribution $\bar{f}(r)$ et correspond donc $\left.{ }^{2}\right)$ à la variance de pareille distribution, on a en définitive

1) La formule (17) est immédiate; quant à la (18), il suffit d'observer que tous les moments depuis l'origine d'une distribution composée satisfont à une relation analogue à la ( 17 ), et que la variance est la différence entre le moment second et le carré du moment premier.

2) Cfr. C. Ginı, Memorie di metodologia statistica, Vol. I, p. 228, Ed. Giuffré, Milano. 


$$
\begin{gathered}
A=A \rho, \\
\bar{\sigma}^{2}=\sigma^{2} \rho+A^{2} \bar{s}^{2} .
\end{gathered}
$$

Sur la base de la distribution des assurés par jours de maladie, qui est contenue dans la Table 3 , et à la distribution des assurés par nombre des cas de la Table I (qui fournit pour $\bar{s}^{2}$ la valeur 0,6703 pour les hommes et la valeur 0,8980 pour les femmes), on a procédé par ailleurs à vérifier les relations (23) et (24). Sur la base des données analytiques du Tableau III qui contient les différentes distributions par nombre de jours des assurés frappés par un seul cas, par deux seuls cas, etc., on a également pu vérifier les relations (I9) et (20) uniquement pour les quatre premières distributions. Les résultats sont les suivants:

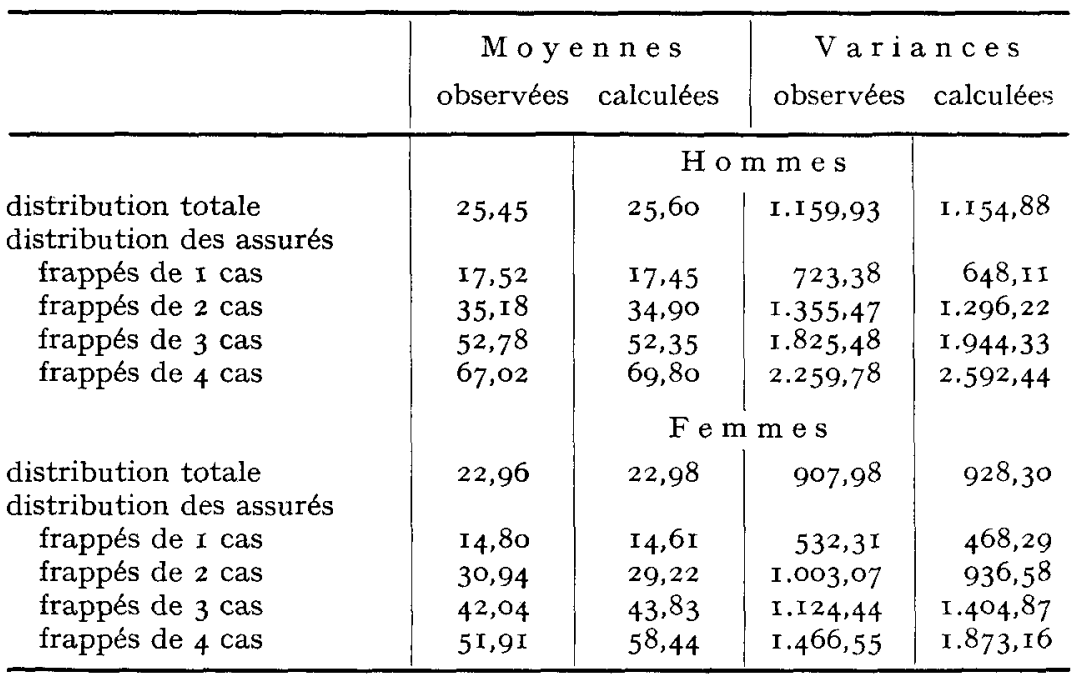

Comme on peut le voir, ces données confirment également assez bien les hypothèses posées, en tenant compte pour les ordres de multiplicité élevés du nombre limité d'observations.

5. Nous nous sommes proposé en dernier lieu la question de savoir si, la fonction $f(r)$ étant connue de même que la fonction $\varphi(u)$, en ses composantes $\varphi_{1}(u)$ et $\varphi_{2}(u)$, il serait possible de déterminer la fonction $\psi(u)$ grâce à une relation plus simple que (I6), même si cette relation est d'une nature seulement expérimentale. 


\section{TABLE 3}

Distribution des assurés par nombre de jours de maladie

\begin{tabular}{|c|c|c|c|c|c|c|c|c|}
\hline \multirow{3}{*}{$\begin{array}{l}\text { Durée } \\
\text { (en } \\
\text { jours) }\end{array}$} & \multicolumn{4}{|c|}{$\mathrm{H} \circ \mathrm{mm} \mathrm{es}$} & \multicolumn{4}{|c|}{ Fem mes } \\
\hline & \multirow{2}{*}{$\begin{array}{c}\text { Données } \\
\text { obser- } \\
\text { vées }\end{array}$} & \multicolumn{3}{|c|}{ Données ajustées (a) } & \multirow{2}{*}{$\begin{array}{c}\text { Données } \\
\text { obser- } \\
\text { vées }\end{array}$} & \multicolumn{3}{|c|}{ Données ajustées (a) } \\
\hline & & distri- & \multicolumn{2}{|c|}{$\begin{array}{l}\text { distributions } \\
\text { partielles }\end{array}$} & & distri- & \multicolumn{2}{|c|}{$\begin{array}{c}\text { distributions } \\
\text { partielles }\end{array}$} \\
\hline$I-5$ & 2.413 & 2.302 & 2.302 & & $2.7^{8} 3$ & 2.445 & 2.445 & \\
\hline 6 - I0 & 2.071 & I.623 & I.604 & I9 & I.929 & I. 590 & I. 522 & 68 \\
\hline II - I 5 & I.243 & 1.030 & 836 & I94 & I.I 58 & I.129 & 723 & 406 \\
\hline $16-20$ & 798 & 848 & $4^{06}$ & $44^{2}$ & 761 & 895 & 3 I 5 & 580 \\
\hline $2 \mathrm{I}-30$ & 1.047 & I. 365 & 274 & $\mathrm{I} .09 \mathrm{I}$ & I.087 & I. $5 \mathrm{I} 8$ & 184 & I.334 \\
\hline $3 I-40$ & 650 & 922 & 55 & 867 & 663 & 990 & 30 & 960 \\
\hline $4 I-60$ & 698 & 977 & I 2 & 965 & $73 I$ & 715 & 5 & 7 IO \\
\hline $6 I-90$ & 487 & 5 I I & I & 510 & 437 & 433 & & 433 \\
\hline $9 \mathrm{I}-\mathrm{I} 2 \mathrm{O}$ & 239 & 230 & & 230 & 244 & 146 & & I 46 \\
\hline I $2 \mathrm{I}-\mathrm{I} 5^{\circ}$ & I37 & 86 & & 86 & $7 \mathrm{I}$ & 60 & & 60 \\
\hline Au délà de & & & & & & & & \\
\hline I5o jours & 217 & 106 & & 106 & I 35 & 79 & & 79 \\
\hline & 10.000 & 10.000 & $5 \cdot 490$ & 4.510 & 10.000 & 10.000 & 5.224 & 4. \\
\hline
\end{tabular}

(a) - Sur la base de la fonction (9) et de ses composantes. Source: INAM - Travailleurs salariés de la province de Rome. Période d'observation: Année 1960.

A cette fin, en rappelant (9) on peut écrire ( $\mathrm{r} 6$ ):

$$
\begin{aligned}
& \psi(u)=\bar{f}(\mathrm{I}) k_{1} \varphi_{1}(u)+\bar{f}(\mathrm{I}) k_{2} \varphi_{2}(u)+\bar{f}(2)[\varphi(u) * \varphi(u)] \\
& +\ldots+\bar{f}(r)[\varphi(u) * \varphi(u) * \ldots * \varphi(u)]+\ldots
\end{aligned}
$$

Admettons que la distribution constituée par tous les termes du second membre de (25) qui suivent le premier puisse être représentée par une fonction du type $\varphi_{2}(u)$, avec des valeurs opportunes des paramètres $a_{2}$ et $p_{2}$, c'est-à-dire:

$$
\psi(u)=\bar{f}(\mathrm{I}) k_{1} \varphi_{1}(u)+\left(\mathrm{I}-\bar{f}(\mathrm{I}) k_{1}\right) \varphi_{2}(u) .
$$

Or $\bar{A}$ et $\bar{\sigma}^{2}$ peuvent être déterminés sur la base des relations (23) et (24) en supposant connues la fonction $\bar{f}(r)$ et la fonction $\varphi(u)$; $A_{1}$ et $\sigma_{1}^{2}$ sont également connus sur la base de la connaissance de la fonction $\varphi_{1}(u)$. D'autre part, $A_{2}$ et $\sigma_{2}^{2}$ peuvent être déter- 
minés, en tenant compte de (I7) et de (I8), au moyen des relations ${ }^{1}$ )

$$
\bar{A}=\bar{f}(\mathrm{I}) k_{1} A_{1}+\left(\mathrm{I}-\bar{f}(\mathrm{I}) k_{1}\right) A_{2}
$$

(28) $\bar{\sigma}^{2}=\bar{f}(\mathrm{I}) k_{1} \sigma_{1}^{2}+\left(\mathrm{I}-\bar{f}(\mathrm{I}) k_{1}\right) \sigma_{2}^{2}+\bar{f}(\mathrm{I}) k_{1}\left(\mathrm{I}-\bar{f}(\mathrm{I}) k_{1}\right)\left(A_{1}-A_{2}\right)^{2}$

et les paramètres $a_{2}$ et $p_{2}$ au moyen des formules

$$
\begin{gathered}
A_{2}=\frac{a_{2}}{p_{2}-2} \\
\sigma^{2}=\frac{a_{2}}{\left(p_{2}-2\right) \sqrt{p_{2}-3}}
\end{gathered}
$$

Nous avons reporté dans la Table 3 les fréquences ajustées au moyen du procédé qui a été décrit: on peut constater que l'adaptation des valeurs calculées aux valeurs observées n'est pas très satisfais ante. Toutefois cette méthode fournit une indication suffisamment significative pour les fins pratiques, dans l'hypothèse posée où on ne disposerait d'aucune donnée relative aux fréquences susdites de la Table 3 .

La moyenne, la variance et les paramètres de la fonction $\varphi_{2}(u)$ ont résulté être les suivants:

$\begin{array}{lrr} & \text { Hommes } & \text { Femmes } \\ A_{2} & 46,54 & 39,9 \mathrm{I} \\ \sigma_{2}{ }^{2} & \mathrm{I} .704,88 & \mathrm{I} \cdot 353,92 \\ a_{2} & \mathrm{10}, 65 & 87,00 \\ p_{2} & 4,27 & 4, \mathrm{I} 8\end{array}$

Il est opportun d'observer que la construction de la fonction $\psi(u)$ permet la détermination des facteurs de réduction rapportés aux assurés, au lieu de ceux rapportés aux cas de maladie, comme parfois cela s'avère nécessaire dans la technique des assurances envisagées.

1) Cfr. l'ouvrage cité en page 53 . 
TABleaU I

Distributions par durée des cas de maladie en distinguant d'après l'ordre dans lequel ils ont eu lieu pour un même assuré

\begin{tabular}{|c|c|c|c|c|c|c|c|c|c|c|}
\hline \multirow[b]{2}{*}{$\begin{array}{l}\text { Durée de la } \\
\text { maladie (en } \\
\text { jours) }\end{array}$} & \multicolumn{10}{|c|}{ Nombre des cas de maladie } \\
\hline &  &  &  & 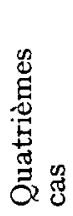 & 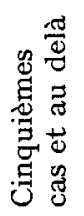 & 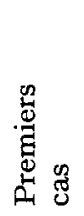 & 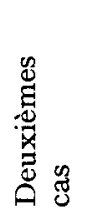 & 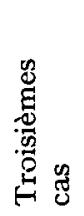 & 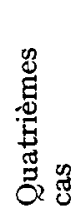 & 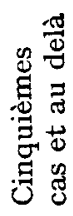 \\
\hline & \multicolumn{5}{|c|}{ Hom mes } & \multicolumn{5}{|c|}{$\mathrm{F}$ e $\mathrm{m} \mathrm{mes}$} \\
\hline $\mathbf{I}$ & I.479 & $43 \mathrm{I}$ & I 47 & 44 & $2 I$ & $43^{8}$ & I 25 & 53 & 27 & 6 \\
\hline 2 & 144 & 42 & I7 & 5 & $\mathbf{r}$ & 25 & 12 & 7 & - & 2 \\
\hline 3 & $\mathbf{I} \cdot 317$ & 442 & $r_{50}$ & $5^{2}$ & 29 & 402 & I 44 & 72 & 30 & $2 \mathrm{I}$ \\
\hline 4 & 363 & I 32 & 36 & I 8 & $\mathbf{I}$ & 90 & 37 & 12 & 8 & 3 \\
\hline 5 & 694 & 208 & 74 & I 4 & 9 & I 76 & 68 & 27 & I3 & 9 \\
\hline 6 & 688 & 218 & 56 & I7 & 6 & 172 & 67 & I6 & 6 & 2 \\
\hline 7 & I.500 & 293 & 95 & 29 & Io & 200 & 78 & 24 & Io & 2 \\
\hline 8 & $6+3$ & I 82 & 57 & 18 & 9 & I32 & $4^{\circ}$ & 20 & 7 & 3 \\
\hline 9 & 429 & I 43 & 50 & I I & Iо & $9 I$ & $3 I$ & I9 & 2 & 2 \\
\hline IO & 406 & Iro & 36 & 8 & 4 & $9 I$ & $4 I$ & I4 & 3 & I \\
\hline II - I5 & 1.554 & 477 & I 75 & 44 & I7 & 376 & 122 & 36 & I I & 9 \\
\hline $16-20$ & $89 \mathrm{I}$ & $27 \mathbf{I}$ & 97 & 25 & 6 & I 85 & 83 & 23 & 8 & 6 \\
\hline $2 I-25$ & 598 & 218 & $6 \mathrm{r}$ & 28 & 6 & $15^{2}$ & 53 & I 7 & 8 & 2 \\
\hline $26-30$ & 433 & $I_{5} \mathrm{I}$ & $5^{\circ}$ & 14 & 2 & 94 & $4^{I}$ & 12 & 3 & I \\
\hline $3^{I}-4^{\circ}$ & 544 & I 86 & 59 & 22 & 7 & I I 2 & 43 & 19 & 2 & 2 \\
\hline $4^{I}-5^{O}$ & 312 & 104 & $4 \mathrm{I}$ & 13 & 2 & 75 & 23 & 9 & 5 & $\mathbf{I}$ \\
\hline $5^{\mathrm{I}}-60$ & I93 & 84 & 27 & 7 & I & 33 & I3 & 6 & I & 3 \\
\hline $6 I-90$ & 346 & 125 & 47 & I6 & 6 & 50 & 24 & 7 & 5 & I \\
\hline $9 \mathrm{I}-\mathrm{I} 20$ & $\mathrm{I}_{42}$ & 69 & 13 & 6 & I & 30 & I3 & 5 & - & 一 \\
\hline$I_{2 I}-I_{50}$ & 85 & I 7 & 6 & 3 & I & 13 & 4 & 2 & 一 & 2 \\
\hline \multicolumn{11}{|l|}{ Au delà de } \\
\hline I 50 jours & 127 & $2 \mathrm{I}$ & 6 & I & - & $\mathbf{I} 7$ & 2 & 2 & 一 & - \\
\hline & 12.363 & 3.924 & 1.300 & 395 & $\mathrm{r}_{49}$ & 2.954 & 1.064 & 402 & I 49 & $7^{8}$ \\
\hline
\end{tabular}




\section{TABLEAU II}

Distribution par durée des cas de maladie en distinguant d'après leur multiplicité par rapport à un même assuré

\begin{tabular}{|c|c|c|c|c|c|c|c|c|c|c|}
\hline \multirow[b]{2}{*}{$\begin{array}{l}\text { Durée de la } \\
\text { maladie (en } \\
\text { jours) }\end{array}$} & \multicolumn{10}{|c|}{ Nombre des cas de maladie } \\
\hline & 㞼 & 晃 & 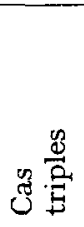 & 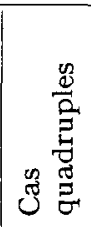 & 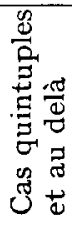 & 苞 & 兽 & $\overbrace{0}^{\mathscr{Q}}$ & 总 & 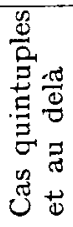 \\
\hline & \multicolumn{5}{|c|}{$\mathrm{H} \circ \mathrm{m} \mathrm{mes}$} & \multicolumn{5}{|c|}{$\mathrm{Fe} \mathrm{m} \mathrm{m} \mathrm{e} \mathrm{s}$} \\
\hline I & 1.013 & 582 & $29 I$ & $\mathrm{I}_{4} 8$ & 88 & 284 & I 68 & 84 & $6 \mathrm{r}$ & $5^{2}$ \\
\hline 2 & $\mathrm{IO}_{5}$ & $5^{I}$ & 34 & $I_{4}$ & 5 & I6 & 9 & 9 & 6 & 6 \\
\hline 3 & 960 & 579 & $24^{8}$ & I 2 I & 82 & 288 & 162 & I I I & 62 & 46 \\
\hline 4 & 256 & 163 & 83 & 35 & I3 & 55 & 44 & 22 & I5 & I 4 \\
\hline 5 & 473 & 272 & 154 & $5^{8}$ & $4^{2}$ & $x 18$ & 75 & $4^{8}$ & 28 & 24 \\
\hline 6 & 447 & 299 & I 59 & 55 & 25 & I 18 & 75 & 36 & 22 & I 2 \\
\hline 7 & 685 & 422 & 196 & 84 & 45 & 122 & IOI & 50 & 26 & I 5 \\
\hline 8 & 404 & 276 & 123 & 44 & 32 & 83 & $5^{6}$ & 36 & 16 & I I \\
\hline 9 & 294 & I 84 & 97 & $4^{8}$ & 20 & 55 & 43 & $3 \mathbf{I}$ & 6 & IO \\
\hline Io & 285 & 160 & 68 & 34 & I 7 & $5^{8}$ & 39 & 30 & 12 & I I \\
\hline II - I 5 & I.028 & 653 & 376 & I 38 & $7^{2}$ & 217 & $\mathbf{I} 79$ & IOI & 33 & 24 \\
\hline $16-20$ & 594 & 369 & 210 & 90 & 27 & 103 & 98 & 55 & 22 & 27 \\
\hline $21-25$ & 415 & $25^{8}$ & I 47 & 67 & 24 & 96 & 66 & 39 & 17 & $\mathrm{r}_{4}$ \\
\hline $26-3^{0}$ & 282 & 199 & I05 & 55 & 9 & 62 & 47 & 24 & I3 & 5 \\
\hline $3 x-40$ & 368 & 242 & I 36 & 55 & I7 & 69 & 60 & 35 & 6 & 8 \\
\hline $4 I-50$ & 225 & 122 & 73 & $4^{I}$ & II & 45 & 34 & 18 & I I & 5 \\
\hline $5 \mathrm{I}-60$ & I25 & 96 & $5^{8}$ & 27 & 6 & 21 & I6 & 8 & 7 & 4 \\
\hline $6 I-90$ & 229 & 167 & $9 \mathrm{r}$ & $3^{6}$ & I7 & 36 & 30 & 9 & 8 & 4 \\
\hline $91-120$ & 86 & 85 & $4^{I}$ & I 5 & 4 & 23 & $\mathrm{I}_{4}$ & 8 & 3 & - \\
\hline $121-150$ & 58 & 34 & I3 & 6 & I & 9 & 5 & 3 & I & 3 \\
\hline \multicolumn{11}{|l|}{ Au delà de } \\
\hline & 107 & 35 & 12 & $\mathbf{I}$ & - & 12 & 3 & 2 & I & 3 \\
\hline & 8.439 & $5.24^{8}$ & $2.7 \mathrm{I} 5$ & I.I 72 & 557 & I. 890 & I.324 & 759 & 376 & 298 \\
\hline
\end{tabular}




\section{TABLEAU III}

Distributions des assurés d'après le nombre des cas et des jours de maladie dont ils ont été frappés au cours de l'année

\begin{tabular}{|c|c|c|c|c|c|c|c|c|c|c|}
\hline \multirow[b]{2}{*}{$\begin{array}{l}\text { Nombre de } \\
\text { jours de } \\
\text { maladie }\end{array}$} & \multicolumn{10}{|c|}{ Nombre des assurés frappés de: } \\
\hline & I cas & 2 cas & 3 cas & 4 cas & $\begin{array}{l}5 \text { cas } \\
\text { et } \\
\text { plus }\end{array}$ & I cas & 2 cas & 3 cas & 4 cas & $\begin{array}{l}5 \text { cas } \\
\text { et } \\
\text { plus }\end{array}$ \\
\hline & \multicolumn{5}{|c|}{$\mathrm{H}$ o $\mathrm{mmes}$} & \multicolumn{5}{|c|}{$\mathrm{Fe} \mathrm{m} \mathrm{m}$ es } \\
\hline $\mathbf{I}$ & I.or 3 & - & 一 & - & - & 284 & - & 一 & - & - \\
\hline 2 & 105 & 72 & - & - & - & I6 & 24 & 一 & - & - \\
\hline 3 & 960 & 2 & $\mathrm{I}_{4}$ & 一 & 一 & 288 & I & 4 & - & - \\
\hline 4 & 256 & 54 & I & 2 & 一 & 55 & 24 & I & 2 & - \\
\hline 5 & 473 & 26 & 5 & - & - & II 8 & 2 & 3 & 一 & - \\
\hline 6 & $44^{8}$ & I I I & 4 & 2 & - & I 18 & 24 & I & - & - \\
\hline 7 & 684 & 50 & 8 & I & $一$ & 122 & I3 & 8 & - & 2 \\
\hline 8 & 404 & $6_{3}$ & 7 & 6 & 2 & 83 & I9 & 2 & 2 & I \\
\hline 9 & 294 & 60 & I7 & I & 2 & 55 & I 5 & 4 & - & 2 \\
\hline IO & 285 & 100 & 6 & 5 & - & $5^{8}$ & $3^{8}$ & $\mathbf{I}$ & 2 & - \\
\hline $\mathrm{II}-\mathrm{I} 5$ & I. .028 & $4^{24}$ & 70 & 12 & 3 & 217 & 97 & I9 & 8 & I \\
\hline $16-20$ & 594 & 314 & 70 & 7 & I & I03 & 85 & 27 & 7 & 3 \\
\hline $2 \mathrm{I}-25$ & $4 \mathrm{I} 5$ & 229 & 77 & 23 & 9 & 96 & 49 & 25 & 7 & 3 \\
\hline $26-30$ & 282 & I 56 & 74 & 25 & 4 & 62 & 43 & 23 & 9 & 4 \\
\hline $3 I-40$ & 368 & 267 & I3I & 24 & I3 & 69 & 74 & 37 & I 2 & 4 \\
\hline $4^{I}-5^{O}$ & 225 & 169 & 86 & 34 & I 2 & 45 & 46 & 28 & 8 & 2 \\
\hline $5 I-60$ & I 25 & YIO & 65 & 27 & Io & $2 \mathrm{I}$ & 35 & $2 I$ & 6 & 4 \\
\hline $6 I-90$ & 229 & 196 & II9 & 43 & I 5 & 36 & 35 & 30 & $I_{4}$ & I 4 \\
\hline $9 I-I 20$ & 86 & 93 & 67 & 33 & I7 & 23 & 20 & 9 & 12 & 8 \\
\hline I $2 \mathrm{I}-\mathrm{I}_{50}$ & $5^{8}$ & 49 & 34 & 23 & 6 & 9 & 5 & 4 & 3 & - \\
\hline Au delà de & & & & & & & & & & \\
\hline I 50 jours & 107 & 79 & 50 & 25 & 8 & 12 & 13 & 6 & 2 & 7 \\
\hline & 8.439 & 2.624 & 905 & 293 & 102 & I. 890 & 662 & 253 & 94 & 55 \\
\hline
\end{tabular}

